\title{
Caracterización de la transmisión de Plasmodium malariae en cuatro regiones colombianas endémicas de malaria
}

\author{
Juana Vergara ${ }^{1}$, Silvia Hurtado ${ }^{1}$, Víctor H. Alvarez ${ }^{2}$, Myriam Arévalo ${ }^{3}$, Sócrates Herrera ${ }^{1}$ \\ 1 Instituto de Inmunología, Universidad del Valle, Cali, Colombia. \\ ${ }^{2}$ Departamento de Epidemiología, Ministerio Nacional de Salud, Bogotá, D.C., Colombia. \\ ${ }^{3}$ Escuela de Bacteriología y Laboratorio Clínico, Universidad del Valle, Cali, Colombia.
}

En Colombia, la detección del número de casos de infección por Plasmodium malariae muestra una distribución porcentual focalizada en la Costa Pacífica, donde alcanza un 95\%, en contraste con el $5 \%$ de casos más esporádicos que se presentan en otras regiones. El departamento del Valle del Cauca aporta el $85 \%$ y el $15 \%$ restante corresponde a los departamentos de Chocó y Cauca. La región de Zacarías aporta el 70\%, seguida por las regiones de Citronela, Puerto Merizalde, Anchicayá, Meseta y Boca Dagua, regiones que conjuntamente contribuyen con el $30 \%$.

En un estudio transversal, realizado en 1997 mediante gota gruesa en la población de Zacarías, se encontró una frecuencia de $9,1 \%$ de casos de $P$. malariae. Se usaron muestras de donantes infectados con gametocitos de $P$. malariae para alimentar mosquitos Anopheles albimanus y reproducir exitosamente el ciclo ex vivo. Se analizaron 242 plasmas obtenidos de individuos de Zacarías y Punta Soldado (Valle) y Pizarro (Chocó) para determinar la presencia de anticuerpos específicos para $P$. malariae. Se encontró que el $9,1 \%$ contenía anticuerpos identificables por la técnica de Elisa contra una preparación cruda de antígeno y que el $42,1 \%$ presentaba anticuerpos contra el péptido sintético (NAAG), derivado de la proteína circumesporozoíto (CS). En 50 muestras analizadas de la región del Bajo Guaviare, se encontró presencia de anticuerpos contra (NAAG) ${ }_{5}$ en el $16 \%$. Los resultados de este estudio muestran una gran delimitación de los casos de la Costa Pacífica, al área de Zacarías, indicando también la posible presencia de este parásito en otras regiones del país.

Palabras clave: malaria, P. malariae, anticuerpos, péptidos, Anopheles.

\section{Characterization of Plasmodium malariae transmission in four malaria endemic regions in Colombia}

The number of cases of Plasmodium malariae in Colombia shows a percent distribution concentrated in the Pacific Coast where it reaches $95 \%$ compared with $5 \%$ of casual cases in other areas of the country. Valle del Cauca region presents $85 \%$ of the cases, while $15 \%$ of the remaining cases correspond to Chocó and Cauca regions. Zacarías has $70 \%$ of the cases, followed by $30 \%$ in the areas of Citronela, Puerto Merizalde, Anchicayá, Meseta and Boca Dagua.

An active search for malaria cases in Zacarías (Buenaventura) using thick smears demonstrated a prevalence of $9.1 \%$ of $P$. malariae cases. Blood samples of gametocyte carriers were used to feed Anopheles albimanus mosquitoes and the reproduction of the sporogonic malaria cycle was successfully achieved. The presence of specific antibodies to P. malariae was analyzed in 242 plasma samples obtained from individuals living in Zacarías and Punta Soldado (Valle), as well as in Pizarro (Chocó). It was found that $9.1 \%$ of the samples had specific antibodies as determined by an Elisa technique using a $P$. malariae crude antigen preparation, whereas $42.1 \%$ of the samples showed specific antibodies when Elisa was performed using the (NAAG) $)_{5}$ synthetic peptide derived from the circumsporozoite (CS) protein. Samples from Bajo Guaviare region displayed a prevalence of $16 \%$ of anti-(NAAG) $)_{5}$ peptide 
antibodies. These results suggest that Zacarías on the Pacific Coast is the main focus of $P$. malariae cases, but also that transmission of this parasite species coud occur in other regions of Colombia not yet reported.

Key words: malaria, P. malariae, antibody, peptides, Anopheles.

Entre las enfermedades transmitidas por vectores, la malaria es la principal causa de enfermedad y muerte a nivel mundial (1). Cerca de 2.400 millones de personas, $40 \%$ del total de la población mundial, vive en áreas maláricas en riesgo de contraer la enfermedad. Se estima que actualmente se producen entre 300 y 500 millones de casos por año, los cuales generan de 1,5 a 2,7 millones de casos letales, principalmente entre niños menores de 5 años y mujeres embarazadas (2). En los últimos 20 años, en América se ha registrado un aumento permanente de la malaria. Mientras en 1974 se informaron cerca de 270 mil casos en una población expuesta a la malaria estimada en 721 millones de personas, el número de casos registrados en 1996 fue más de 1 millón en 2.400 millones de personas expuestas. Además de este incremento significativo, se conoce que existe un importante subregistro de casos, hecho que implica que el número real de casos es aún mayor.

El país con mayor prevalencia de malaria en la región es Brasil, donde anualmente se registran alrededor de 500 mil casos (3). En Colombia, en 1997 , se informaron 180.970 casos de los cuales $63,3 \%$ fueron causados por Plasmodium vivax, $36,3 \%$ por Plasmodium falciparum, $0,3 \%$ se registraron como infecciones mixtas y solamente el $0,1 \%$ fueron causadas por Plasmodium malariae. A pesar de su amplia distribución, en Colombia la malaria tiene mayor prevalencia en las regiones de Antioquia y de la Costa Pacífica (4). Sin embargo, estudios recientes sobre la transmisión de malaria en la Costa Pacífica indican que la región aporta alrededor del $20 \%$ de la incidencia nacional anual discriminada en $67,4 \%$ por $P$. falciparum, $28,5 \%$ por $P$. vivax y $4,1 \%$ por P. malariae (5).

Estas cifras revisten gran importancia epidemiológica debido a que la población expuesta

Correspondencia:

Juana Vergara, Apartado aéreo 25574, Cali, Colombia

Recibido: 20/02/00; aceptado: 05/02/01 en el Litoral Pacífico representa solamente alrededor del 2,2\% de la población nacional (6), y a que la proporción de casos de $P$. malariae, a pesar de su baja incidencia nacional, representa en el Pacífico cerca del $95 \%$ de los casos. Se considera que $P$. malariae es el más antiguo de los parásitos de la malaria humana (7) y que debido a la prolongada interacción parásito/hospedero, la adaptación del parásito al hombre es más evolucionada que la de las otras especies, lo cual genera un menor daño y una sintomatología más benigna (fiebre cuartana) (8). Sin embargo, a pesar de no inducir mortalidad por enferrnedad aguda, la persistencia crónica del parásito en el organismo causa gradualmente una hipertrofia esplénica mayor que la producida por las otras especies de Plasmodium. Luego de varios años de infección recurrente, esta especie produce un síndrome conocido como 'necrosis cuartana' consistente en anemia, leucopenia y depósito de pigmento malárico en los órganos. Esta condición clínica se acompaña de daño renal, caracterizado por albuminuria, nefritis parenquimatosa crónica, edema generalizado y, finalmente, cambios intersticiales con alteración cardiovascular asociados con las recrudescencias periódicas de la enfermedad (9).

La respuesta humoral inducida por $P$. malariae se caracteriza por una significativa estimulación policlonal de linfocitos B con una abundante producción de anticuerpos inespecíficos, en tanto que sólo una pequeña parte de ellos tiene alta especificidad por el parásito (10).

Su baja prevalencia en Colombia y la localizada distribución geográfica hacen de la malaria por $P$. malariae una entidad a la que se le presta poca atención médica y epidemiológica a pesar de que podría estar causando patología renal crónica, no diagnosticada en las poblaciones endémicas. Ante la inexistencia de información específica sobre esta especie en Colombia, se realizó el presente trabajo en el cual se describen algunas características epidemiológicas e inmunológicas 
en cuatro poblaciones colombianas: Zacarías y Punta Soldado (Valle), Pizarro (Chocó) y Bajo Guaviare (Guaviare). Estos resultados sugieren que la transmisión de P. malariae podría no ser tan localizada como se consideraba previamente.

\section{Materiales y métodos}

\section{Areas de estudio, censo de población y de vivienda}

El estudio se realizó en 3 localidades de la Costa Pacífica (Zacarías, Pizarro y Punta Soldado) y 6 localidades de la región del Bajo Guaviare, seleccionadas por ser sitios de permanente transmisión malárica, en los cuales el Instituto de Inmunología del Valle adelanta estudios epidemiológicos. Las localidades de la Costa Pacífica tenían antecedentes de transmisión de $P$. malariae de acuerdo con los registros del Servicio de Erradicación de la Malaria, mientras que no existen antecedentes de transmisión en el Guaviare, razón por la cual se consideraron estas comunidades como población control.

La población de Zacarías cuenta con un total de 752 habitantes, $43 \%$ hombres y $57 \%$ mujeres. La distribución porcentual de personas por edad es como sigue: menores de un año, $5,7 \%$; de 1 a 5 años, $14,7 \%$; de 6 a 15 años, 28,6\%; de 16 a 45 años, 35\%, y mayores de 45 años, $37 \%$; tiene 82 viviendas con un promedio de 9 personas por vivienda. Punta Soldado está compuesto por 432 habitantes, $48 \%$ hombres y $52 \%$ mujeres, distribuidos así: 0 a 5 años, 25\%; 6 a 20 años, $20 \%$; 21 a $45,35 \%$, y mayores de $45,20 \%$; cuenta con 74 viviendas con un promedio de 6 personas por casa. Pizarro, área urbana del municipio del
Bajo Baudó, consta de 5.017 habitantes, 51\% hombres y $49 \%$ mujeres. Su distribución por grupos de edad es: 0 a 5 años, $15 \%$; 6 a 20 años, $26 \%$; 21 a 45 años, $42 \%$, y mayores de 45 años, $17 \%$. Cuenta con 642 viviendas. Bajo Guaviare consta de 1.859 habitantes divididos en 6 grupos étnicos diferenciados entre sí por rasgos culturales y esencialmente por la lengua que hablan: Guahibo $(27,4 \%)$, Piapoco $(22,7 \%)$, Piaroa $(20,2 \%)$, Puinave $(12,3 \%)$, Curripaco $(9,4 \%)$ y Cubeo $(0,7 \%)$. La población mestiza $(7,3 \%)$ está compuesta por colonizadores del interior del país.

\section{Plasmodium malariae en Colombia y áreas de estudio}

De acuerdo con el registro de casos de infección por P. malariae suministrados por el Ministerio de Salud, durante los años 1990 a 1997 se observó un promedio de 53 casos anuales, con un mínimo de 32 y un máximo de 92 casos/año, de los cuales aproximadamente $80 \%$ fueron informados en el departamento del Valle del Cauca y originados principalmente en el municipio de Buenaventura (4)(cuadros 1 y 2). $95 \%$ del total de casos anuales por P. malariae a nivel nacional lo aporta la Costa Pacífica; de ellos, $85 \%$ proviene del departamento del Valle del Cauca, de los cuales Zacarías aporta alrededor de $70 \%$, Punta Soldado 0 a 3\%, Pizarro 0 a $5 \%$ y las demás localidades aparecen menos definidas. Las localidades estudiadas del Bajo Guaviare no presentan registro de malaria por $P$. malariae.

\section{Estudio transversal de malaria}

Debido a la transmisión continua de malaria y a su cercanía a Buenaventura y Cali, Zacarías fue

Cuadro 1. Casos de malaria en Colombia. Comparación entre el número total de casos/año y la proporción de casos de $P$. malariae en Colombia y en la Costa Pacífica.

\begin{tabular}{|c|c|c|c|c|c|c|}
\hline \multirow{3}{*}{ Año } & \multicolumn{3}{|c|}{ Colombia } & \multicolumn{3}{|c|}{ Costa Pacífica } \\
\hline & \multirow{2}{*}{$\begin{array}{l}\text { Total casos } \\
\text { de malaria }\end{array}$} & \multicolumn{2}{|c|}{ P. malariae } & \multirow{2}{*}{$\begin{array}{l}\text { Total casos } \\
\text { de malaria }\end{array}$} & \multicolumn{2}{|c|}{ P. malariae } \\
\hline & & $\mathbf{n}$ & $\%$ & & $\mathrm{n}$ & $\%$ \\
\hline 1990 & 99.489 & 67 & 0,06 & 25.903 & 65 & 0,30 \\
\hline 1991 & 184.156 & 92 & 0,04 & 40.154 & 92 & 0,20 \\
\hline 1992 & 184.023 & 37 & 0,02 & 45.955 & 37 & 0,10 \\
\hline 1993 & 129.377 & 53 & 0,04 & 23.730 & 45 & 0,20 \\
\hline 1994 & 127.218 & 40 & 0,03 & 16.899 & 39 & 0,20 \\
\hline 1995 & 187.082 & 41 & 0,02 & 22.001 & 39 & 0,20 \\
\hline 1996 & 131.438 & 46 & 0,03 & 14.523 & 45 & 0,30 \\
\hline 1997 & 180,970 & 52 & 0,03 & 11.579 & 52 & 0,40 \\
\hline
\end{tabular}


Cuadro 2. Distribución de casos de P. malariae en Colombia. Casos de P. malariae durante los años 1990-97.

\begin{tabular}{lccccc}
\hline Año & Colombia & \multicolumn{4}{c}{ Costa Pacífica } \\
\cline { 3 - 6 } & & Valle & Chocó & Cauca & Otros $^{*}$ \\
\hline 1990 & 67 & 51 & 8 & 6 & 2 \\
1991 & 92 & 84 & 6 & 2 & 0 \\
1992 & 37 & 27 & 3 & 7 & 0 \\
1993 & 53 & 25 & 16 & 4 & 8 \\
1994 & 40 & 31 & 7 & 2 & 0 \\
1995 & 41 & 32 & 5 & 2 & 2 \\
1996 & 46 & 45 & 0 & 0 & 1 \\
1997 & 105 & 96 & 0 & 9 & 0 \\
\hline
\end{tabular}

Meta, Guaviare y Tolima

seleccionada hace varios años como población estudio. Observaciones preliminares indicaron que una alta concentración de casos de malaria $(4,1 \%)$ de esta localidad se debían a P. malariae (5); así, se realizó la revisión de registros de malaria del Ministerio de Salud durante el período 1990-1997 y se adelantó un estudio de corte transversal en la localidad. En octubre de 1997 se realizó un censo de población y de vivienda y, a la vez, la búsqueda activa de infección malárica por la técnica de gota gruesa (11), casos que fueron evaluados a través de la observación microscópica en 100 o más campos con 2 lecturas independientes. La parasitemia se calculó considerando el número de parásitos contados en presencia de 200 leucocitos y expresados como parásito/ $\mu$ l. Igualmente, se construyó una base de datos sobre los aspectos socioeconómicos de la población.

\section{Obtención y procesamiento de las muestras}

El cálculo del tamaño de la muestra se realizó teniendo en cuenta el tamaño de la población de las áreas de estudio (Zacarías, Punta Soldado, Pizarro y Bajo Guaviare), la prevalencia esperada de casos de malaria y el resultado más alejado de la prevalencia de malaria por arriba y por abajo usando el programa Epilnfo 6. El cálculo indicó que, para un nivel de confianza de $99 \%$, con una prevalencia de $30 \%$, el tamaño de la muestra mínima por estudiar era de 118 en Zacarías, 48 en Punta Soldado, 50 para Pizarro y 46 para Bajo Guaviare. El análisis serológico se realizó utilizando 292 muestras de plasma de voluntarios de las regiones de Zacarías $(n=142)$, Punta Soldado $(n=50)$, Pizarro $(n=50)$ y Bajo Guaviare $(n=50)$; las muestras se tomaron por venopunción braquial en tubos que contenían heparina como anticoagulante. Parte de los plasmas utilizados pertenecen al banco de muestras del Instituto de Inmunología, utilizados en otros estudios $(5,13,28)$. Las muestras se separaron en plasma y glóbulos rojos por centrifugación durante 10 minutos a $1.500 \mathrm{rpm}$. El plasma obtenido se mantuvo a $-70^{\circ} \mathrm{C}$ hasta su evaluación.

\section{Estudios serológicos}

Los anticuerpos específicos contra P. malariae se determinaron por una prueba de inmunofluorescencia indirecta (IFI) y por la técnica de Elisa. Las láminas para IFI se prepararon a partir de sangre de un paciente infectado con $P$. malariae con un recuento de 150 parásitos en 200 leucocitos (aproximadamente, 6.000 parásitos/ $\mu$ l). La sangre con $0,2 \%$ de parásito/ $\mu$ l se lavó 3 veces mediante centrifugación con bufferfosfato (PBS). Luego de descartar la capa de leucocitos, los glóbulos rojos parasitados se resuspendieron en PBS con $2 \%$ de albúmina bovina hasta obtener un hematocrito de $1 \%$. Cada pozo de la lámina de fluorescencia se cubrió con $10 \mu \mathrm{l}$ de la suspensión y se almacenó a $-70{ }^{\circ} \mathrm{C}$ hasta el momento de su utilización.

La prueba IFI se realizó con los sueros humanos diluidos seriadamente en PBS a partir de 1:20. Como segundo anticuerpo, se utilizó un suero de conejo anti-lgG humana, marcada con fluoresceína (aH-lgG-FITC) y diluida en PBS 1:100, siguiendo la técnica descrita previamente (12).

La prueba de Elisa se realizó utilizando como antígeno el péptido (NAAG) ${ }_{5}$ correspondiente a un epítope $B$ inmunodominante derivado de la región central repetitiva de la proteína CS de P. malariae (13). Este péptido se sintetizó utilizando la técnica F-moc (14), en un equipo Advanced Chemtech $396 \mathrm{MBS}$. El péptido se diluyó a una concentración de $1 \mu \mathrm{g} / \mathrm{ml}$ y se agregó a las microplacas de 96 pozos $(100 \mu \mathrm{l} /$ pozo) para después incubarlo durante 16 horas; luego, cada pozo fue saturado durante 1 hora con una solución de PBS que contenía 0,05\% Tween-20 y $5 \%$ de leche sin lípidos (fat free milk). El plasma diluido 1:200 en PBS se agregó a cada pozo por 1 hora y se incubó a temperatura ambiente en cámara húmeda. 
Después de 4 lavadas con PBS + 0,05\% Tween20 , las microplacas se incubaron una vez más por término de 1 hora con anti-lgG humana marcada con fosfatasa alcalina diluida 1:1000 en PBS-Tween-leche. Luego de 4 lavadas adicionales, se agregó una solución sustrato que contenía $1 \mathrm{mg} / \mathrm{ml}$ de $\mathrm{p}$-nitrofenilfosfato diluido en buffer de dietanolamina. Luego de una incubación de 30 minutos a temperatura ambiente, la densidad óptica de la reacción se leyó a $405 \mathrm{~nm}$. Se estableció un punto de corte sumando 3 veces la media al valor obtenido de la lectura de cada muestra sin péptido.

\section{Infectividad de mosquitos Anopheles albimanus a P. malariae}

El mosquito Anopheles albimanus es una de las especies vectoras de malaria más ampliamente distribuidas en el continente americano. Este mosquito es considerado el vector más importante de la Costa Pacífica colombiana. Para determinar la susceptibilidad de $A$. albimanus a la infección por $P$. malariae se realizaron experimentos de infección artificial de mosquitos previamente colonizados (15), utilizando sangre parasitada proveniente de pacientes de la región de Zacarías. La alimentación infectiva se realizó en jaulas con 300 a 500 mosquitos hembra, utilizando tubos alimentadores de vidrio cubiertos con membranas artificiales. Los mosquitos fueron expuestos a infección con muestras de sangre provenientes de pacientes infectados con $P$. malariae, las cuales fueron mantenidas a $37^{\circ} \mathrm{C}$ para evitar la exflagelación del parásito. Los mosquitos fueron expuestos durante 20 minutos a infección con muestras de sangre que presentaron rangos entre 4.000 y 6.000 parásito $/ \mathrm{ml}$; inmediatamente después, se retiraron las hembras que no se alimentaron. Entre 10 y 25 hembras infectadas de cada lote se disecaron el día 12 después de la infección para detectar ooquistes en sus estómagos. Entre los días 15 y 20 después de la alimentación, las glándulas salivares se disecaron para recolectar lcs esporozoítos almacenados en sus lóbulos distales y demostrar así la susceptibilidad a la infección por P. malariae. El número de esporozoítos se estimó microscópicamente, realizando el recuento en un hemocitómetro con el objetivo de 40X.

\section{Resultados}

\section{Prevalencia de Plasmodium malariae}

La presencia de malaria en la comunidad de Zacarías se evaluó en los meses de febrero, mayo, agosto y octubre de 1997 mediante una búsqueda activa por la técnica de gota gruesa. Se examinaron 206 gotas gruesas, 66 (32\%) de las cuales resultaron positivas. La relación infección/ especie fue de $60,6 \%$ para $P$. falciparum, $30,3 \%$ para $P$. vivax y $9,1 \%$ para $P$. malariae (cuadro 3 ). El rango de parasitemia observada para $P$. falciparum y $P$. vivax estuvo entre 200 y 11.400 parásitos $/ \mu \mathrm{l}$, mientras que para $P$. malariae fue de 80 a 1.600 parásitos/ $\mu$ l. En estudios previos de muestreo masivo en poblaciones de la Costa Pacífica, el número de casos de malaria por $P$. malariae encontrados en estas zonas fue de $4,1 \%$ (5) y en el presente estudio alcanzó un valor de $9,1 \%$ del total de casos informados en Zacarías.

\section{Respuesta humoral a malaria}

La presencia de anticuerpos anti-esporozoítos medidos por la técnica de Elisa, utilizando como antígeno péptido sintético (NAAG) ${ }_{5}$, demostró una prevalencia de $62,0 \%$ para Zacarías, $12 \%$ en Pizarro, $30 \%$ en Punta Soldado y $16 \%$ para la región del Bajo Guaviare en la población adulta estudiada (cuadro 4). Además, se determinó la presencia de anticuerpos anti-CS de $P$. vivax y $P$. falciparum en 142 plasmas de la región de Zacarías, de los cuales 3 plasmas respondieron a múltiples péptidos de las diferentes especies estudiadas, mientras 8 presentaron anticuerpos dirigidos exclusivamente hacia el péptido (NAAG) . $_{5}$. El $37 \%$ y el $76 \%$ de los plasmas respondieron a $P$. falciparum y $P$. vivax, respectivamente. Los anticuerpos contra la forma sanguínea se analizaron solamente para los sueros de Zacarías, utilizando la técnica IFI. El análisis demostró una

Cuadro 3. Distribución porcentual por especies de Plasmodium circulantes en Colombia.

\begin{tabular}{lcccc}
\hline Especie & Nacional & $\begin{array}{c}\text { Costa } \\
\text { Pacífica }\end{array}$ & Zacarías & $\begin{array}{c}\text { Bajo } \\
\text { Guaviare }\end{array}$ \\
\hline P. malariae & 0,03 & 0,3 & 9,1 & - \\
P. vivax & 69,85 & 34,5 & 30,3 & 63,9 \\
P. falciparum & 30,12 & 65,1 & 60,6 & 36,1 \\
\hline
\end{tabular}


Cuadro 4. Prevalencia de anticuerpos anti-esporozoítos en adultos de cuatro localidades de área endémica a malaria.

\begin{tabular}{lcrr}
\hline Región & N & \multicolumn{2}{c}{ Positivas } \\
& & \# & $\%$ \\
\hline Zacarías & 76 & 47 & 62 \\
Punta Soldado & 50 & 15 & 30 \\
Pizarro & 50 & 6 & 12 \\
Bajo Guaviare & 50 & 8 & 16 \\
\hline
\end{tabular}

$\mathrm{N}$ : número de muestras analizadas

Muestras positivas por Elisa

respuesta positiva en $10,5 \%$ de los plasmas estudiados, con títulos que variaron entre un 1:20 y 1:320, mientras que los anticuerpos medidos por la técnica de Elisa con antígeno crudo mostraron una positividad de $9,1 \%$ (cuadro 5).

\section{Susceptibilidad de A. albimanus a infección por P. malariae}

Estudios entomológicos previos (16), realizados con especímenes de anofelinos silvestres de la Costa Pacífica, indican que el principal vector de malaria en esta región es $A$. albimanus; $\sin$ embargo, los índices esporozoíticos para $P$. falciparum y $P$. vivax han sido muy bajos. Debido a la baja incidencia de la infección por P. malariae y al limitado número de mosquitos utilizado en dichos estudios no ha sido posible identificar mosquitos infectados con $P$. malariae en la naturaleza. Teniendo en cuenta que $A$. albimanus es la especie más abundante en la región y aprovechando la disponibilidad de esta especie colonizada, se probó la infectividad de aislados silvestres de P. malariae. Las gotas gruesas de 5

Cuadro 5. Presencia de anticuerpos anti- $P$. malariae en Zacarías, Buenaventura.

\begin{tabular}{|c|c|c|c|c|}
\hline \multirow[t]{2}{*}{ Edad } & \multirow[t]{2}{*}{ Población ${ }^{1}$} & \multirow[t]{2}{*}{$\mathrm{GG}^{2}$} & \multicolumn{2}{|c|}{ Anticuerpos } \\
\hline & & & $\mathrm{Spz}{ }^{3}$ & $\mathrm{FS}^{4}$ \\
\hline $0-1$ & 6 & 2 & 0 & 0 \\
\hline $2-5$ & 19 & 15 & 3 & 0 \\
\hline $6-15$ & 41 & 14 & 9 & 4 \\
\hline $16-45$ & 60 & 23 & 28 & 6 \\
\hline$>45$ & 16 & 12 & 19 & 3 \\
\hline
\end{tabular}

${ }_{1}^{1}$ Número de individuos estudiados por grupo de edad

${ }^{2}$ GG: número de casos positivos por gota gruesa

${ }^{3}$ Anticuerpos anti-esporozoíto (Spz) medidos por Elisa usando como antígeno el péptido (NAAG)

${ }^{4}$ Anticuerpos anti-formas sanguíneas (FS) medidos por Elisa con antígeno crudo donantes provenientes de Zacarías mostraron gametocitemias entre 0,1 y $0,2 \%$. La susceptibilidad a la infección fue evidenciada por la presencia de ooquistes en los estómagos de los mosquitos el día 12 después de la infección y esporozoítos en sus glándulas salivares desde el día 15, en todos los lotes expuestos. Los mosquitos examinados mostraron un número variable de 152.000 a 1'725.000 esporozoítos en $27,2 \%$ de los lotes expuestos, lo cual demuestra la capacidad de esta especie para el desarrollo del ciclo esporogónico completo.

\section{Discusión}

El análisis realizado en este estudio indica que el número de casos de malaria registrados por el Ministerio de Salud en el país y en la Costa Pacífica cada año es relativamente estable. A pesar de que $P$. vivax es la especie predominante a nivel nacional $(65 \%)$, en la Costa Pacífica el principal agente causante de morbimortalidad por malaria es $P$. falciparum. La menor incidencia de P.vivax se debe probablemente a la alta prevalencia de individuos portadores del fenotipo Duffy negativo (Fy-) resistentes a la infección por esta especie. Al igual que en el oeste de Africa, P. malariae es de baja transmisión comparada con $P$. falciparum y $P$. vivax (17). Esta baja transmisión de infección en la región puede atribuirse a cambios climáticos, al desarrollo de la inmunidad en la población local y a la disponibilidad de drogas antimaláricas, al igual que en la India y Suramérica, donde la baja prevalencia de $P$. malariae se atribuye a la inmunidad y a cambios climáticos $(18,19)$. P. malariae a nivel nacional presenta un promedio anual de solamente $0,03 \%$. Sin embargo, el promedio en la Costa Pacífica se eleva a 0,3\%, lo que obedece a que esta región aporta cerca del $95 \%$ del total de casos de P. malariae del país. Se observó una concentración de los casos en el departamento del Valle del Cauca, seguido del Chocó, Cauca y algunos casos esporádicos en otros departamentos. Nuestra búsqueda permitió identificar a la localidad de Zacarías, ubicada en la zona rural del municipio de Buenaventura, como el foco principal de transmisión de P. malariae en Colombia con un aporte del $70 \%$ de los casos de $P$. malariae informado por el departamento del Valle del Cauca. Esta localidad presentó una 
frecuencia relativa de $9,1 \%$ de infección por $P$. malariae y prevalencia de anticuerpos antiesporozoítos de $36,6 \%$ por IFI, utilizando esporozoítos, y de $41,5 \%$ contra el péptido (NAAG) 5 por Elisa. Los anticuerpos contra formas sanguíneas del parásito fueron de $10,5 \%$ por IFI. Estos resultados sugieren más alta inmunogenicidad del péptido (NAAG), similar a la demostrada en ratones de experimentación inmunizados con este péptido (20).

La prevalencia de anticuerpos en las diferentes localidades de la Costa Pacífica estudiadas confirma la mayor transmisión en la localidad de Zacarías y una prevalencia de anticuerpos similar en otras regiones de la Costa Pacífica. Guaviare, considerado hasta ahora exento de transmisión de $P$. malariae, podría, al igual que otras regiones del país, tener transmisión de esta especie; sin embargo, no se puede descartar la existencia de epítopes compartidos entre $P$. malariae y otros microorganismos, particularmente de las otras especies de Plasmodium (21). Alternativamente, podría haber una reacción cruzada entre variantes naturales de epítopes CS(21). Algunos determinantes antigénicos son compartidos por diferentes moléculas, especialmente si éstas son moléculas similares de especies relativas. En estos casos, algunos anticuerpos son inducidos en respuesta a un antígeno dado, pero se puede unir a otros antígenos, presentándose una reacción cruzada. Una definición de reacción cruzada puede también ser asignada a la unión del anticuerpo a una molécula diferente al determinante antigénico que la indujo. Una muy interesante observación es la reacción cruzada del anticuerpo P. malariae con el esporozoíto de malaria de simio ( $P$. brasilianum), la cual indica la completa o próxima identidad de epítopes de la proteína CS de las dos especies (22). El trabajo demuestra que, al igual que en el viejo mundo, el $P$. malariae es una especie focalizada y que posiblemente se transmite fuera de los sitios registrados por el Ministerio de Salud. Sin embargo, esta observación requiere mayor estudio. Como ocurre con las otras especies de malaria humana, las personas en edad productiva presentaron mayor presencia de anticuerpos probablemente debido a una mayor exposición al parásito.
La capacidad de A. albimanus de desarrollar el ciclo esporogónico completo de P. malariae en condiciones experimentales podría tener gran importancia epidemiológica. A. albimanus es una especie de gran prevalencia en esta región y ha sido previamente incriminada como vector de $P$. falciparum y $P$. vivax. En el Instituto de Inmunología se está evaluando actualmente la capacidad vectorial de este mosquito para $P$. malariae en experimentos con primates del género Aotus.

Finalmente, el análisis de los registros médicos del hospital de Buenaventura no evidenció la presencia de casos de patología renal que puedan asociarse con malaria por P. malariae; se plantea la incógnita de si las patologías renales informadas son ajenas a $P$. malariae y si los casos de malaria por $P$. malariae en niños conllevan las complicaciones descritas previamente en regiones altamente endémicas para este parásito.

Ante la focalización de la transmisión de esta especie, podría pensarse en la viabilidad de una estrategia de erradicación del foco descrito, cuyo propósito fundamental sea el de reducir no sólo la incidencia de malaria, sino principalmente la remoción de los factores específicos del riesgo existente en el área. Una vez identificado el foco de transmisión, se deben adaptar las estrategias recomendadas por la OMS en 1998 a las condiciones locales, entre las cuales cabe señalar las siguientes: 1) involucrar a la comunidad, realizando charlas educativas de como prevenir y tratar la malaria; 2) implementar estrategias de saneamiento peridomiciliario, realizando limpieza de la maleza, correcta disposición de las basuras y drenaje de lagunas y charcas; 3) realizar prácticas de control biológico en depósitos de agua con Bacillus (sphearicus o B. thuringiensis) o con peces; 4) proporcionar un adecuado y oportuno tratamiento quimioprofiláctico con drogas gameticidas a personas infectadas para controlar la transmisión de la infección; 5) fortalecer estas estrategias mediante el rociamiento y fumigación de las viviendas y áreas peridomiciliarias, y 6) establecer el uso rutinario de toldillos impregnados con insecticidas para reducir el riesgo de infección, disminuyendo el número de picaduras de 
mosquitos infectados y la carga del parásito asociada con morbilidad y mortalidad por la infección malárica (23).

Las posibles desventajas de esta estrategia son la aparición de resistencia a las drogas antimaláricas en humanos y la resistencia a insecticidas en mosquitos $(24,25)$. Además, la presencia de la malaria se asocia al deterioro social y a las precarias condiciones económicas, así como a la ausencia de servicios de salud, migración humana y deterioro del medio ambiente.

\section{Agradecimientos}

Queremos resaltar la activa participación de la comunidad de Zacarías, principalmente la de sus promotores de salud Sixto Tulio Arboleda y Samuel Mondragón. Igualmente, la participación del doctor Alejandro Hoyos, director del Programa de Enfermedades Tropicales (P.E.T. Buenaventura) y el apoyo técnico del químico John Jairo Valencia en la síntesis del péptido (NAAG) 5 .

\section{Referencias}

1. World Health Organization. Twelfth program report of the UNPD/World Bank/WHO special program for research and training in tropical diseases (TDR). Bull World Health Organ 1996;71:17-24.

2. Organización Panamericana de la Salud. Las condiciones de la salud en las Américas. Publicación científica 1996;2:549.

3. Curado I, Duarte AM, Lal A, Oliveira SG, Kloetzel JK. Antibodies anti-bloodstream and circumsporozoite antigens (Plasmodium vivax and Plasmodium malariae) $P$. brasilium) in areas of very low malaria endemicity in Brazil. Mem Inst Oswaldo Cruz 1997;92:235-43.

4. Ministerio Nacional de Salud. Departamento de Epidemiología, Campañas Directas. Registro de casos de malaria a nivel nacional y por departamento 19901997.

5. González JM, Olano V, Vergara J, Arévalo-Herrera M, Carrasquilla G, Herrera S, et al. Unstable, low level transmission of malaria on the Colombian Pacific Coast. Ann Trop Med Parasitol 1997;91:349-58.

6. Departamento Administrativo Nacional de Estadística, DANE. Censo poblacional a nivel nacional y por departamentos 1993 .

7. Garnham PCC. Malaria parasite and other Haemosporidia. En: Quartan malaria parasite. First edition. Oxford: Blackwell Scientific Publications 1966; p.257-81.

8. Taylor-Robinson AW. Glomerulonephritis and nephrotic syndrome in Plasmodium chabaudi chabaudi: a potentiall murine model of chronic $P$. malariae infection. Ann Trop Med Parasitol 1996;90:635-7.

9. Ward PA, Kibukamusoke JW. Evidence for soluble immune complexes in the pathogenesis of the glomerulonephritis of quartan malaria. Lancet 1969;1: 283-5.

10. Allison AC, Hendrickse RG, Edington GM, Houba V, Petris S de, Adeniyi A. Immune complexes in the nephrotic syndrome of African children. Lancet 1969;1: 1232-8.

11. Makler MT, Palmer CJ, Ager AL. A review of practical techniques for the diagnosis of malaria. Ann Trop Med Parasitol 1998;92:419-33.

12. Voller A, O'Neill P. Inmunofluorescence method suitable for large scale application. Bull World Health Org $1971 ; 45: 524-9$.

13. Arévalo-Herrera M, Roggero MA, González JM, Vergara J, Corradin G, López JA, et al. Mapping and comparison of the B-cell epitopes recognized on the Plasmodium vivax circumsporozoite protein by immune Colombians and immunized Aotus monkeys. Ann Trop Med Parasitol 1998;92:539-51.

14. Lloyd-Willians P, Albericio F, Giralt E. Solid-phase peptide synthesis. En: Chemical approaches to the synthesis of peptides and proteins. Boca Ratón: CRC Press 1997; p.19-92.

15. Hurtado S, Salas ML, Romero JF, Zapata JC, Ortiz $\mathrm{H}$, Arévalo-Herrera $\mathbf{M}$, et al. Regular production of infective sporozoites of Plasmodium falciparum and $P$. vivax in laboratory-bred Anopheles albimanus. Ann Trop Med Parasitol 1997;91:49-60.

16. Herrera S, Suárez MF, Sánchez GL, Quiñones ML, Herrera MA. Uso de la técnica inmunoradiométrica (IRMA) en Anopheles de Colombia para la identificación de esporozoitos de Plasmodium. Colombia Médica 1987; 18:2-6.

17. Boudin C, Robert V, Verhave JP, Carnevale P, Ambroise-Thomas P. Plasmodium falciparum and $P$. malariae epidemiology in a West African village. Bull World Health Org 1991;69:199-205.

18. Panda R, Verma KVS, Rahman SJ. Present status of Plasmodium malariae infection in Bastar district (MP). J Com Dis 1990;22:185-90.

19. De Arruda ME, Aragaki C, Gagliardi F, Haile RW. A seroprevalence and descriptive epidemiological study of malaria among Indian tribes of the Amazon basin of Brazil. Ann Trop Med Parasitol 1996;90:135-43.

20. Del Giudice G, Tougne Ch, Louis JA, Lambert PH, Bianchi E, Bonelli F, et al. A multiple antigen peptide from the repetitive sequence of the Plasmodium malariae circumsporozoite protein induces a specific antibody response in mice of various $\mathrm{H}-2$ haplotypes. Eur $\mathrm{J}$ Immunol 1990;20:1619-22. 
21. Zevering $\mathbf{Y}$, Khamboonruang $\mathrm{C}$, Good MF. Human and murine T-cell responses to allelic forms of a malaria circumsporozoites protein epitope support a polyvalent vaccine strategy. Immunology 1998;94:445-54.

22. Cochrane AH, Collins WE, Nussenzweig RS. Monoclonal antibody identifies circumsporozoite protein of Plasmodium malariae and detects a common epitope on Plasmodium brasilianum sporozoites. Infec Imm 1984;45:592-5.
23. Rojas W, Peñaranda F, Echavarría M. Strategies for malaria control in Colombia. Parasitology Today 1992; 8:141-5.

24. WHO. Vector resitance to pesticides. Fifteenth report of the WHO expert committee on vector biology and control. Geneva: World Health Organization; 1992.

25. Adubofour KO. Drug resistance in malaria: a review of the West African situation. J Natl Med Assoc 1992; 84:1025-9. 